\title{
Bioreducible nanocapsules for folic acid- assisted targeting and effective tumor-specific chemotherapy
}

This article was published in the following Dove Press journal: International Journal of Nanomedicine

\section{Qiangying $\mathrm{Yi}^{\mathrm{l}}$ \\ Jin $\mathrm{Ma}^{\mathrm{l}}$ \\ Ke Kang' \\ Zhongwei Gu ${ }^{1,2}$}

'National Engineering Research Center for Biomaterials, Sichuan University, Chengdu, ${ }^{2}$ College of Materials Science and Engineering, Nanjing Tech University, Nanjing, P.R. China
Correspondence: Zhongwei Gu National Engineering Research Center for Biomaterials, Sichuan University, 29 Wangjiang Road, Chengdu 610064, P.R. China

Tel/fax $+8628854 \mid 0653$

Email zwgu@scu.edu.cn
Introduction: Increasing demands in precise control over delivery and functionalization of therapeutic agents for tumor-specific chemotherapy have led to a rapid development in nanocarriers. Herein, we report a nanocapsule (NC) system for tumor-oriented drug delivery and effective tumor therapy.

Materials and methods: Functionalized hyaluronan is utilized to build up the NC shells, in which bioreduction cleavable sites, targeting ligand folic acid (FA), and zwitterionic tentacles are integrated.

Results: The hollow NCs obtained ( $\sim 50 \mathrm{~nm}$ in diameter) showed well-defined spherical shell structures with a shell thickness of $\sim 8 \mathrm{~nm}$. These specially designed NCs (doxorubicin [DOX]/ FA-Z-NCs) with high drug encapsulation content exhibited good biocompatibility in vitro and fast intracellular drug release behavior mediated by intracellular glutathione.

Conclusion: Cellular uptake tests demonstrated rapid uptake of these functionalized NCs and effective escape from endosomes. Antitumor efficacy of the DOX/FA-Z-NCs was confirmed by the significant tumor growth inhibition effect as well as greatly reduced side effects, in contrast with those of the free drug DOX hydrochloride.

Keywords: drug delivery, active targeting, zwitterionic poly(carboxybetaine methacrylate), doxorubicin

\section{Introduction}

Chemotherapy has been one of the most commonly used strategies for tumor treatment. Although improved survival rate of patients has been achieved, application of chemotherapeutics in clinical use is seriously restricted by its adverse effects due to poor solubility, high toxicity, lack of specificity, and so on. ${ }^{1}$ Drug delivery systems have, therefore, been developed to cope with the challenges. Various carriers in the form of micelles, liposomes, assemblies, drug conjugates, nanoparticles, and so on are widely used for drug delivery. Their developments from discovery to applications have been well investigated and summarized. ${ }^{2}$ However, potential disadvantages, such as carriers' instability in biologic fluids ${ }^{3}$ and short circulation period ${ }^{4}$ in the blood stream, have often limited their uses in vivo.

As spherical hollow interiors surrounded by steady shells, "capsules" have served as multifunctional systems by offering tremendous opportunities to integrate active/ functional components and various guest substances into one entity. ${ }^{5}$ Microcapsules have been considered as highly promising platforms for encapsulation of drugs ${ }^{6} /$ biomacromolecules (eg, DNA and cell growth factor $)^{7,8}$ and controlled release in cells triggered by either intracellular stimuli ${ }^{9}$ or remote energy fields. ${ }^{10,11}$ Besides, by 
utilizing the functionalized microcapsules, manipulation of cell movements, ${ }^{12}$ visualization of intracellular drug traffic, ${ }^{13}$ and transportation in living body ${ }^{14}$ could be feasible. Nanocapsules, compared with the microcapsules, are smaller in size. Regarding the clinically used nanomedicines in tumor therapy, nanocapsules with hydrodynamic diameter in the range of $30-200 \mathrm{~nm}$ are preferred to primarily realize accumulation of the chemotherapeutic agents in tumor sites through enhanced permeability and retention, even though the enhanced permeability and retention effect in clinical application is failing. ${ }^{15}$ Due to the fancy architectures and unmet degree of multifunctionalities, nanocapsules should have been widely used to provide solutions for current scientific problems in the biomedical domain. However, to the best of our knowledge, only a very few research studies focusing on preparation of nanocapsules for potential biomedical uses have been reported. One of the major causes limiting their potential applications might be attributed to the difficulty to fabricate steady nanoscaled $(<300 \mathrm{~nm})$ hollow shell structures without aggregation.

An alternative approach to develop well-dispersed stable nanocarriers could be addressed by fabrication of hydrogel nanoparticles (nanogels). ${ }^{16}$ The crosslinked three-dimensional hydrophilic networks of this kind of nanoparticles were filled with $\mathrm{H}_{2} \mathrm{O}$ molecules. Consequently, aggregation of these nanogels in aqueous solutions could be greatly avoided. On this basis, the strategy of using the hydrogel-like structures to build up nanocapsules would become feasible. A successful example has been demonstrated by Wauthoz et al who utilized the hydrogel nanocapsules with encapsulated prodrug gemcitabine for targeting lymph nodes and combating local mediastinal metastases. ${ }^{17}$ On the other hand, the intelligent nanocapsules should be capable of responding to external stimuli to realize drug encapsulation and release by changing their intrinsic properties, such as shell permeability, volume, water affinity, and so on. In previous studies, temperaturesensitive nanocapsules with crosslinked shells composed of triblock copolymers and corresponding complementary polymers were studied. ${ }^{18,19}$ The authors focused only on their in vitro bioevaluations and found that these nanocapsules enabled successful in vitro cytosol-specific delivery of the therapeutic agents through temperature-mediated volume transitions (shrinkage or swelling). However, it should be noted that, despite the strict demand for accurate control over synthesis of the triblock copolymers, the volume changes were achieved by the so-called "cold-shock" treatment. In other words, a significant temperature decrease to $20^{\circ} \mathrm{C}$ or even lower (to $15^{\circ} \mathrm{C}$ ) was basically required to achieve the volume changes aimed at. For further applications in biomedical areas, especially as chemotherapy in clinical applications, payload release induced by this dramatic temperature decrease may seem unlikely.

In this work, we propose a simple yet efficient method to prepare the hydrogel-like nanocapsules and provide construction strategies to govern their transportation and responses toward tumor microenvironments for tumor-specific drug release. Briefly, the natural biomacromolecule hyaluronic acid (HA) derivative was chosen as the only building material for nanocapsules, and oil-in-water $(\mathrm{O} / \mathrm{W})$ emulsion approach was established to generate nanocaspule shells. This $\mathrm{O} / \mathrm{W}$ emulsion could realize nanocapsule shell construction, stabilization, as well as drug encapsulation in one step, saving time and simplifying the operation process. Formed nanocapsules would benefit from the intrinsic properties of HA, such as good biocompatibility, high binding affinity to HA receptors (ie, CD44) in tumors, ${ }^{20}$ and availability for further chemical modification. ${ }^{21}$

To achieve high extent of drug accumulation at tumor sites, ligand-based active targeting of nanocarrier/chemotherapeutic agent to cancer cells would be one of the most popular approaches. Unlike the nonspecific delivery, targeting ligand conducted delivery acts in a hugely selective fashion with enhanced efficacy against solid tumors and other circulating tumor cells. ${ }^{22}$ Among those targeting ligands studied, folic acid (FA) represents a type of smallmolecular ligand, and it was reported that FA showed a high affinity to the glycosylphosphatidylinositol-linked folate receptor, which was overexpressed (100-300 times higher) at the surface of many types of cancer cell lines including ovarian, breast, and lung cancer cells, compared to very limited distribution observed in normal tissues. ${ }^{23}$ Strategically, incorporating FA into our nanocaspules would greatly enhance their cellular uptake through FA receptor-mediated endocytosis. In a previous work, FA was directly conjugated to the surface of nanocarriers or decorated on the hydrophilic tentacles of the nanocarriers. ${ }^{24}$ The most commonly used tentacle in biomedical areas is polyethylene glycol (PEG) for its ability to repel nonspecific protein absorption and to enable a long circulation period in the bloodstream. Although the utilization of PEG (also known as PEGylation) has been widely adopted to decorate nanocarriers, recent studies have shown that PEGylation has some critical shortcomings, the most typical of which is the accelerated blood clearance phenomenon. ${ }^{25}$ In previous studies, $\mathrm{we}^{26}$ and other researchers ${ }^{27}$ have demonstrated that the zwitterionic polymers were suitable alternatives of PEG, showing benefits due to their good biocompatibility, antifouling properties, $\mathrm{pH}$ responses, and so on. Moreover, terminal groups of the 
zwitterions (ie, $-\mathrm{COOH},-\mathrm{NH}_{2}$ ) could offer facile active sites for further modification, for example, the targeting ligand conjugation. Specifically, in this work, the zwitterionic poly(carboxybetaine methacrylate) was chosen to construct hydrophilic tentacles on nanocapsules to ensure prolonged circulation time in blood and also to realize conjugation of FA molecules for active targeting.

Herein, we propose a new strategy to fabricate the hydrogellike nanocapsules for drug delivery, as shown in Scheme 1. The functional HA nanocapsules possessed the following
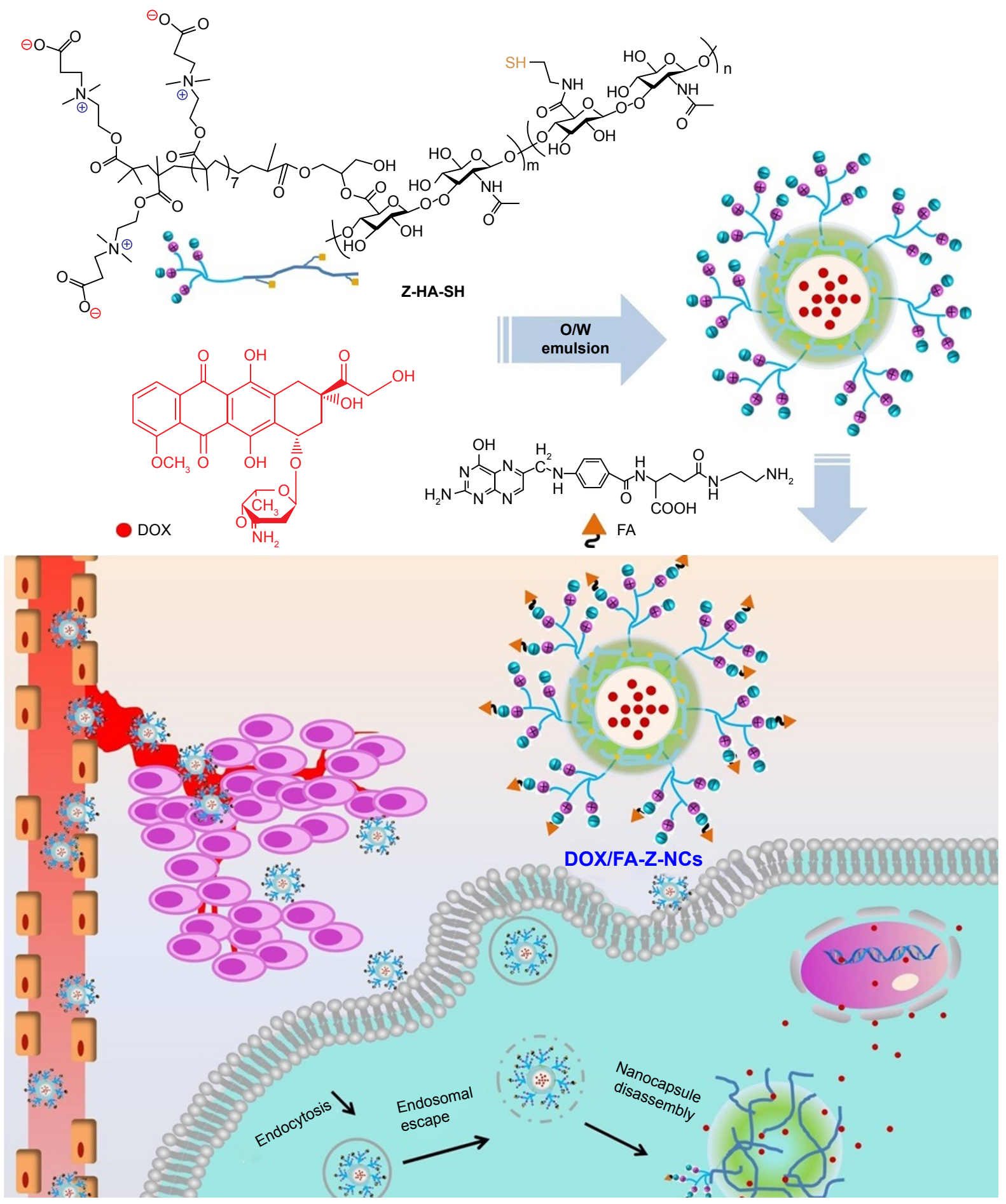

Scheme I Active targeting of the nanocapsules and intracellular drug trafficking.

Abbreviations: DOX, doxorubicin; FA, folic acid; HA, hyaluronic acid; NCs, nanocapsules; O/W, oil in water. 
attractive features. Firstly, the nanocapsule shells were built up with the signal component HA derivative. Secondly, the hollow interiors formed would provide large cavities for encapsulation of antitumor drugs without any drug conjugation or chemical modification process to the drug molecules. Thirdly, the targeting ligand FA was connected to the terminal of zwitterionic tentacles, offering antifouling property as well as active targeting toward the tumors. Specifically, doxorubicin (DOX), one of the most commonly used antitumor therapeutic agents and a fluorescent substance, was studied in this work as a model drug. To verify the construction strategy proposed in our work, in vitro and in vivo bioevaluations as well as potential antitumor efficacy was studied and examined in detail.

\section{Materials and methods Materials}

HA (molecular weight $[\mathrm{Mw}]=100 \mathrm{kDa}$ ) was obtained from Sunlidabio Ltd (Nanjing, China). Mouse breast tumor cell line (4T1) was obtained from the Chinese Academy of Science Cell Bank for Type Culture Collection (Shanghai, China). FA, doxorubicin hydrochloride (DOX.HCl), ethylene diamine, glycidyl methacrylate (GM), reduced L-glutathione (GSH), 2,2-dimethoxy-2-phenylacetophenone, cystamine dihydrochloride, triethylamine, 1-hydroxybenzotriazole, (1-ethyl-3-[dimethylamino]propyl)carbodiimide hydrochloride, $N, N^{\prime}$-dicyclohexylcarbodiimide, $N$-hydroxysuccinimide, and other chemicals were purchased from Sigma-Aldrich (St Louis, MO, USA).

\section{Esterification of cystamine-conjugated HA (HA-Cys)}

HA-Cys was prepared by reacting the $N$-hydroxysuccinimideactivated carboxyl groups of HA with cystamine dihydrochloride. ${ }^{28}$ To introduce the zwitterionic segment onto the HA chains, methacrylate groups were added to HA-Cys to yield GM-conjugated HA-Cys (GM-HA-Cys; Scheme S1). ${ }^{29}$ Later, the disulfide ( $-\mathrm{S}-\mathrm{S}-$ ) bonds of cystamine components were cleaved after being treated with an excess amount of dithiothreitol. The final product obtained (denoted as GMHA-SH) was dialyzed against water, freeze-dried, and stored at $4^{\circ} \mathrm{C}$ for further study.

\section{Zwitterion-functionalized HA derivatives}

The zwitterions were used to functionalize HA by grafting carboxybetaine methacrylate (CBMA) to GM-HA-SH. First, the macroinitiator polyCBMA-Br (pCBMA-Br) was synthesized through the atom-transfer radical polymerization of the monomer CBMA together with initiator (ethyl $\alpha$-bromoisobutyrate), ligand $(1,1,4,7,10,10$-hexamethyltrie thylenetetramine [HMTETA]), and catalyst (CuBr). ${ }^{26}$ After purification and freeze-drying, the obtained macroinitiator was reacted with catalyst $(\mathrm{CuBr})$ and the ligand (HMTETA), and then GM-HA-SH was added. The molar ratio of GM and CBMA was controlled at 1:10. After several purge cycles, the nitrogen-protected mixture was placed in an oil bath for a $24 \mathrm{~h}$ reaction. After completion of the reaction, the raw product was dialyzed against phosphate buffer saline (PBS): N,N-Dimethylformamide (DMF) (9:1, v:v), filtered (0.22 $\mu \mathrm{m}$ Millex-GP filter, EMD Millipore, Billerica, MA, USA) to remove insoluble components, and freeze-dried. The product obtained was denoted as Z-HA-SH.

\section{Nanocapsule preparation and drug encapsulation}

Preparation of HA nanocapsules (NCs) was performed by using the $\mathrm{O} / \mathrm{W}$ emulsion method. ${ }^{28} \mathrm{FA}$ functionalization was achieved by binding the aminated FA ( $\underline{\text { Scheme } \mathrm{S} 2})^{30,31}$ molecules to the terminal carboxyl groups of zwitterionic tentacles of prepared nanocapsules through carbodiimide reaction. For ultraviolet (UV)-visible (vis) measurements, the zwitterionic nanocapsules without DOX encapsulation (Z-NCs) were prepared first and then the aminated FA was introduced. The molar ratio of the aminated FA was proposed to be $10 \%$ of the terminal carboxyl groups of zwitterions. After completion, the product was dialyzed against water, freeze-dried, and denoted as FA-Z-NCs. UV-vis spectrum was applied to confirm the successful introduction of the FA. The prepared nanocapsules with encapsulated DOX were named as DOX/ FA-Z-NCs.

\section{Characterizations of the nanocapsules}

Dynamic light scattering (DLS) measurements were applied to detect the size distribution and zeta potential of prepared nanocapsules with a Zetasizer Nano-ZS90 (Malvern Instrument, Malvern, UK). DOX encapsulation content was confirmed by a fluorescence spectrophotometer (F-7000; Hitachi Ltd, Tokyo, Japan) with $\lambda_{\text {ex }}=480 \mathrm{~nm}$ and $\lambda_{\text {em }}=589 \mathrm{~nm}$. Transmission electron microscopy (Tecnai GF20S-TWIN, FEI Corp., Hillsboro, OR, USA) and scanning electron microscopy (Hitachi S-4800) were used to acquire morphologies of the prepared nanocapsules. UV absorption spectra of the FA-Z-NCs suspension, Z-NCs suspension, and FA solutions in quartz cuvettes were measured with a UV-vis spectrophotometer (TU1901; Persee, Beijing, China) and the absorbance in the range 200-500 nm was recorded.

Quantification of encapsulated DOX content was carried out using an F7000 fluorescence spectrometer (Hitachi Ltd). Generally, freeze-dried HA nanocapsules containing DOX 
(DOX/FA-Z-NCs and DOX/Z-NCs) were resuspended in $\mathrm{H}_{2} \mathrm{O}$ $\operatorname{DMF}(1: 5, \mathrm{v} / \mathrm{v})$ and GSH was added; they were ultrasonically treated and then related fluorescent signals were recorded with the maximum excitation wavelength at $480 \mathrm{~nm}$ and emission wavelength at $588 \mathrm{~nm}$.

\section{Drug release}

GSH-induced drug release from the HA nanocapsules was monitored by detecting fluorescent signals of the released DOX molecules with the excitation wavelength $\lambda_{\text {ex }}=480 \mathrm{~nm}$ and the emission wavelength $\lambda_{\mathrm{em}}=589 \mathrm{~nm}$. One portion of the DOX-containing nanocapsules (DOX/FA-Z-NCs or DOX/Z$\mathrm{NCs}$ ) in a dialysis bag was immersed in 20 portions of the incubation medium (0.01 M PBS, $\mathrm{pH} 7.4$ ) with varied GSH concentrations (GSH 0, GSH $2.8 \mu \mathrm{M}$, and GSH $10 \mathrm{mM}$ ) at $37^{\circ} \mathrm{C}$ with shaking. At set time points, one portion of the incubation medium was collected for the test and one portion of the corresponding incubation medium was added back to replenish the reservoir. The DOX content determination was, therefore, accomplished by referring to a series of DOX solutions with known concentrations $(0-10 \mu \mathrm{g} / \mathrm{mL})$.

\section{Tumor cell culture and animals}

All the tests involving cells and animals were conducted in Sichuan University; animal care and treatment were guided and approved by the animal research ethics committee of Sichuan University. Mouse breast cancer cells (4T1) were cultured in DMEM culture medium supplemented with $10 \%$ fetal bovine serum $\left(\mathrm{GIBCO}^{\circledR}\right.$ Life Technologies, Carlsbad, CA, USA), $1 \%$ penicillin, and streptomycin at $37^{\circ} \mathrm{C}$ with $5 \%$ $\mathrm{CO}_{2}$. BALB/c nude mice were provided by Dossy Biological Technology Co., Ltd. (Chengdu, China). These mice were allowed to acclimate for 1 week before experimental treatments.

\section{Cell viability assay}

The cell counting kit-8 (CCK-8) assay was used to determine cell viability of the $4 \mathrm{~T} 1$ cells co-cultured with DOX-containing formulations. In brief, the 4T1 cells seeded on 96-well plates were treated with DOX.HCl, DOX/FA-Z-NC, and DOX/ZNCs with varied DOX content $(0.001-100 \mu \mathrm{g} / \mathrm{mL})$, and cocultured for $36 \mathrm{~h}$. CCK-8 assay was carried out according to the manufacturer's instructions. 4T1 cells treated with hollow capsules (without DOX encapsulation) were used to test biocompatibility of the nanocapsules.

\section{Flow cytometric analysis and endosomal escape}

To detect cellular uptake, 4T1 cells $\left(3 \times 10^{4}\right)$ seeded in 12-well plates were co-cultured with the DOX-containing formulations
(DOX-HCl, DOX/FA-Z-NC, and DOX/Z-NCs) for a certain period of time. After removal of the culture medium, the cells were treated with trypsin, washed with PBS, and enhanced cellular uptake behavior was recorded by flow cytometry (BD Accuri C6; Becton Dickinson, Franklin Lakes, NJ, USA). For endosomal escape studies, LysoTracker ${ }^{\circledR}$ Green DND-26 (Thermo Fisher Scientific, Waltham, MA, USA) was used to label the acidic compartments of 4T1 cells. Then, these cells were co-cultured with the DOX-containing formulations for a certain period of time and observed by confocal laser scanning microscopy (CLSM; Leica TCP SP5; Leica, Wetzlar, Germany).

\section{In vivo antitumor efficacy}

To investigate the in vivo antitumor efficacy, the subcutaneous tumor model was established to simplify the study. Four-week-old female BALC/c mice (body weight 17-19 g) were injected with $4 \mathrm{~T} 1$ cells $\left(5 \times 10^{5}\right.$ cells in $50 \mu \mathrm{L}$ PBS $)$ to establish subcutaneous tumors under their left arms. When the tumor size grew up to about $180 \mathrm{~mm}^{3}$, these tumorbearing BALC/c mice were randomly divided into four groups $(n>5)$ and treated with $200 \mu \mathrm{L}$ of saline or one of the other three DOX-containing formulations $(\mathrm{DOX} \cdot \mathrm{HCl}$, DOX/FA-Z-NCs, DOX/Z-NCs) through their tail veins. The injected DOX dosage was normalized to be $3 \mathrm{mg} / \mathrm{kg}$. The treatment was carried out every 4 days, and five injections in total were administered. Related body weight and tumor volume of each mouse was carefully recorded every 2 days. The tumor volume $(V)$ was expressed as $V=\mathrm{L} \times \mathrm{W}^{2} / 2$ , where $\mathrm{L}$ and $\mathrm{W}$ represent the measured tumor length and tumor width, respectively. After the experimental period, all the tumor-bearing BALC/c mice were sacrificed. The major organs including heart, liver, spleen, lung, kidney, and tumor were carefully excised and used for histologic and immunohistochemical studies.

\section{Results and discussion Chemical modifications of HA}

The cystamine substitution degree of HA-Cys was calculated according to the ${ }^{1} \mathrm{H}$ nuclear magnetic resonance (NMR) spectrum of HA-Cys by comparing the normalized integrals of acetamido moiety ( $\delta=1.91 \mathrm{ppm})$ of HA and methylene ( $\delta=2.90$ ppm, $-\mathrm{S}-\mathrm{S}-\mathrm{CH}_{2}-\mathrm{CH}_{2}-$ ) of cystamine (Figure $\mathrm{S} 1$ ). The substitution degree was determined to be about $50 \%$. Similarly, the ${ }^{1} \mathrm{H}$ NMR spectrum of GM-HA-SH was used to verify the methacrylation degree (Figure S2). Rationalizing and comparing the integrations of single protons from the methyl groups $\left(\delta=1.89 \mathrm{ppm},-\mathrm{CO}-\mathrm{C}\left(=\mathrm{CH}_{2}\right)-\mathrm{CH}_{3}\right)$ on methacrylate (of GM) against the methyl groups of 
HA $\left(\delta=1.96 \mathrm{ppm},-\mathrm{NH}-\mathrm{CO}-\mathrm{CH}_{3}\right)^{32}$ or the ethylenic bonds $\left(\delta=5.25\right.$ and $\left.5.13 \mathrm{ppm},-\mathrm{CO}-\mathrm{C}\left(=\mathrm{CH}_{2}\right)-\mathrm{CH}_{3}\right)$ on acrylate (of GM segments) against the methyl groups $\left(\delta=1.96 \mathrm{ppm},-\mathrm{NH}-\mathrm{CO}-\mathrm{CH}_{3}\right)$ of $\mathrm{HA},{ }^{33}$ the methacrylation was determined to be $\sim 10 \%$.

${ }^{1} \mathrm{H}-\mathrm{NMR}$ spectrum $\left(\mathrm{D}_{2} \mathrm{O}, 400 \mathrm{MHz}\right.$, Bruker) confirmed successful introduction of the zwitterionic segments onto the HA (Z-HA-SH) chains (Figure S3). Generally, the enhanced peak intensities or broadened peaks represented corresponding chemical shifts of protons of the pCBMA, although these peaks were mostly covered by the signals of other protons of the GM-HA-SH: $\delta=0.89-1.06\left(-\mathrm{CH}_{2}-\mathrm{C}\left(\mathrm{CH}_{3}\right)\right.$ $\mathrm{COO}-), 2.61-2.67\left(-\mathrm{CH}_{2}-\mathrm{COOH}\right), 2.87-2.90\left(-\mathrm{N}^{+}\left(\mathrm{CH}_{3}\right)_{2}-\right)$, $3.31\left(-\mathrm{N}^{+}\left(\mathrm{CH}_{3}\right)_{2}-\mathrm{CH}_{2}-\right), 3.40-3.42\left(-\mathrm{CH}_{2}-\mathrm{N}^{+}\left(\mathrm{CH}_{3}\right)_{2}-\right)$, and $4.31\left(-\mathrm{CO}-\mathrm{CH}_{2}-\right)$. Comparing the ${ }^{1} \mathrm{H}-\mathrm{NMR}$ spectra of GM-HA-SH and Z-HA-SH, it was deduced that there were about nine carboxybetaine methacrylate units grafted onto one esterified HA unit.
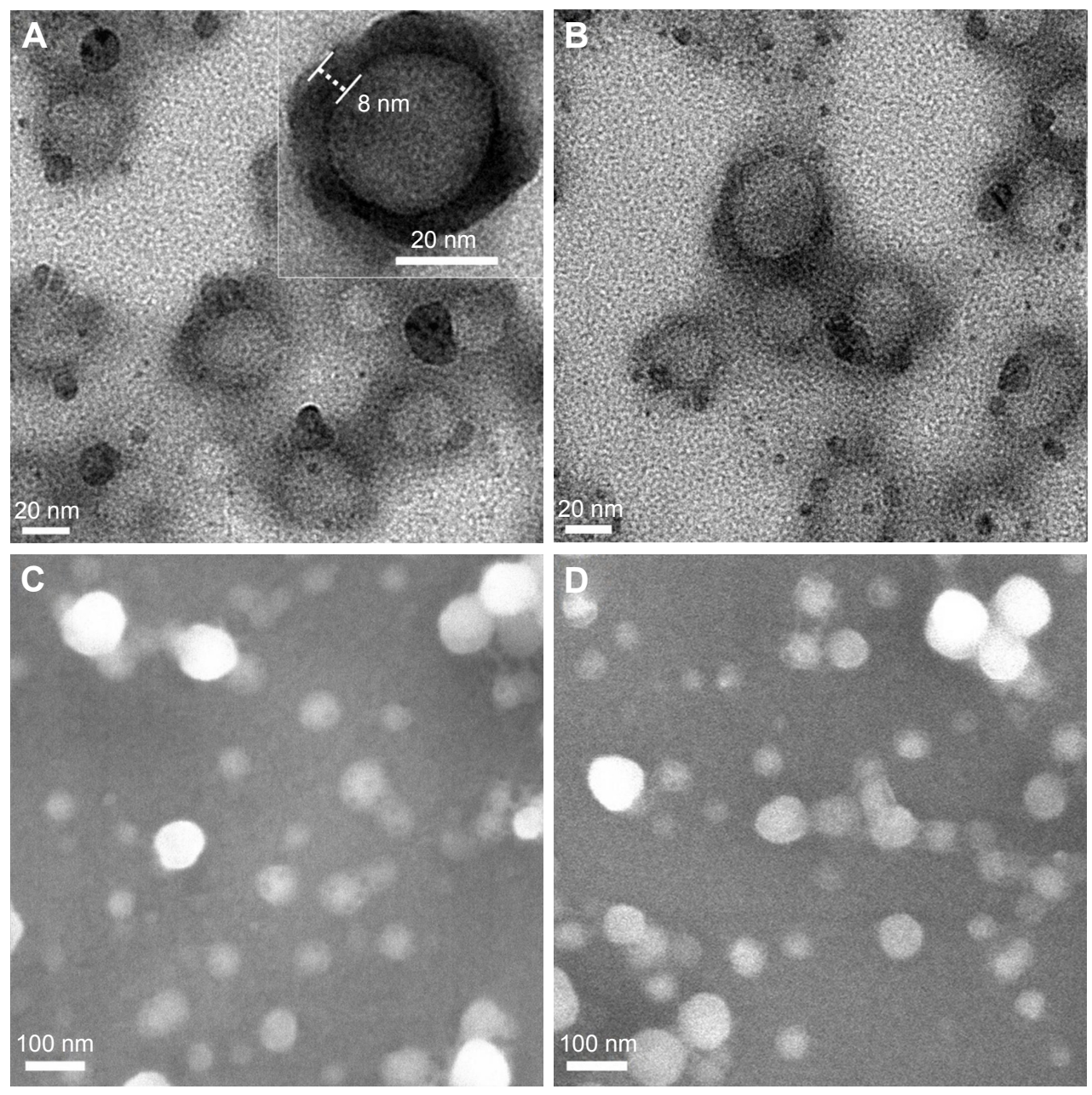

Figure I TEM (top panel) and SEM (bottom panel) images of DOX/FA-Z-NCs (A, C) and DOX/Z-NCs (B, D).

Note: The inset in (A) shows a typical example of the hollow nanocapsule (FA-Z-NC) in the magnified view.

Abbreviations: DOX, doxorubicin; FA, folic acid; NCs, nanocapsules; SEM, scanning electron microscopy; TEM, transmission electron microscopy.

\section{Characterization of the nanocapsule} systems

Introduction of the aminated FA (its ${ }^{1} \mathrm{H}$ NMR spectrum is shown in Figure S4) onto the nanocapsules was monitored by UV-vis spectroscopy (Figure S5). An absorption peak centered at $283 \mathrm{~nm}$ was attributed to the UV absorption of the FA. For the zwitterion-functionalized hollow capsules (Z-NCs), no absorbance could be detected in this location. On the contrary, an obvious absorbance peak at $282 \mathrm{~nm}$ was detected for the nanocapsules with FA conjugation (FA-Z$\mathrm{NCs}$ ). These results provided semi-quantitative evidence to confirm the successful decoration of the FA on the Z-NCs. Referring to the standard calibration curve, which was obtained from the UV-vis spectra of aminated FA solutions with known concentrations, the molar ratio of the conjugated FA to terminal carboxyl groups was $\sim 1: 4.6$. DOX encapsulation content was found to be $11 \%$ for DOX/FA-Z-NCs and $13 \%$ for $\mathrm{DOX} / \mathrm{Z}-\mathrm{NCs}$. 
Electron microscopy images of the prepared drugencapsulating nanocapsules were recorded (Figure 1). After evaporation of chloroform, the hollow cavities formed can be visualized under transmission electron microscopic observation directly (Figure 1A and B). Average diameters were found to be $48.83 \pm 6.14 \mathrm{~nm}$ for DOX/FA-Z-NCs and $46.00 \pm 6.09 \mathrm{~nm}$ for DOX/Z-NCs. A typical example of the hollow nanocapsule (FA-Z-NC) is demonstrated (Figure 1A, inset), in which the shell thickness was detected to be about $8 \mathrm{~nm}$. As for the scanning electron microscopic measurements, these nanocapsules were observed as spheres (Figure 1C and D). Average diameters were measured to be $55.52 \pm 11.88 \mathrm{~nm}$ for DOX/FA-Z-NCs and $51.65 \pm 10.02$ $\mathrm{nm}$ for DOX/Z-NCs. Resuspending these lyophilized nanocapsules in water led to a remarkable increase in capsule size. The hydrodynamic diameters increased to $189.2 \mathrm{~nm}$ (polydispersity index [PDI] 0.167) for DOX/FA-Z-NCs and $193.1 \mathrm{~nm}$ (PDI 0.161) for DOX/Z-NCs (Figure S6). The capsule swelling phenomenon was supported by the fact that the crosslinked nanocapsule shells could adsorb a large amount of water to form hydrogel-like structures. ${ }^{31}$ It was obvious that, no significant difference either in morphology or in the average diameter between these two nanocapsule systems could be observed by using the electron microscopy and DLS characterizations, demonstrating that conjunction of FA did not cause obvious changes to the nanocapsules.

\section{GSH-induced capsule disassembly and drug release}

In vivo reduction conditions are normally used to undermine the stability of the drug carriers and, thus, to govern release of their payloads. Cellular GSH is one of the mainly studied factors. GSH concentration is at a much higher level (mM) in the cytosol and subcellular compartments. However, it is quite low $(\mu \mathrm{M})$ in plasma and tumor-surrounding environments because of the fast degradation by enzymes. ${ }^{34}$ In this work, we investigated the nanocapsule stability in three different buffers which could be applied to mimic related GSH conditions inside/outside the tumor cells and in plasma, respectively, as follows: 1) PBS containing $10 \mathrm{mM} \mathrm{GSH}$; 2) PBS containing $2.8 \mu \mathrm{M}$ GSH; and 3) PBS containing no GSH. In general, stability of the DOX/FA-Z-NCs and DOX/Z-NCs was largely dependent on treated GSH concentration. In $10 \mathrm{mM}$ GSH buffer, they showed a swelling and disassembly process over time (Figure S7). After $12 \mathrm{~h}$, outlines of the nanocapsules could be discerned; after $24 \mathrm{~h}$, no intact shell-like structure could be found. This GSHinduced nanocapsule disassembly offered a fancy way to release encapsulated DOX inside the cells rapidly. As shown in Figure 2, the DOX release triggered by GSH was investigated. At high GSH level, for instance, at $10 \mathrm{mM}$, DOX release rate was remarkably fast as compared with that of the other two groups (GSH $2.8 \mu \mathrm{M}$ and GSH 0). At the GSH level of $10 \mathrm{mM}$, most of the DOX was rapidly released from the nanocapsules by the first $12 \mathrm{~h}$. Specifically, the cumulative DOX release was found to be $69.49 \%$ for DOX/FA-Z-NCs and $65.81 \%$ for DOX/Z-NCs when the incubation time reached $12 \mathrm{~h}$. After that, the DOX release rate slowed down slightly and gradually approached equilibrium. For the DOX/ FA-Z-NCs, DOX release was detected as $81.91 \%$ at $24 \mathrm{~h}$, and then the accumulated DOX reached a stable level of $\sim 82 \%$ during the period from 36 to $72 \mathrm{~h}$, indicating that the DOX
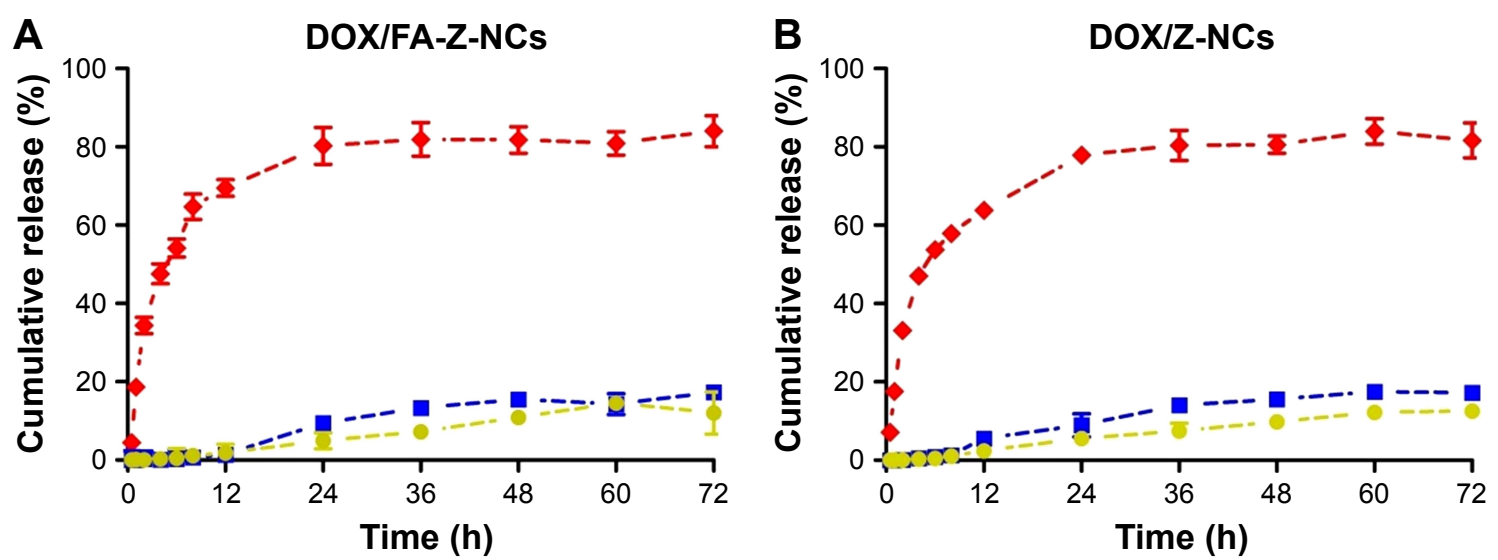

Figure 2 DOX release from nanocapsules triggered by various reduction conditions. Notes: (A) DOX/FA-Z-NCs; (B) DOX/Z-NCs.

Abbreviations: DOX, doxorubicin; FA, folic acid; GSH, glutathione; NCs, nanocapsules; PBS, phosphate buffer saline. 
release was almost unchanged over this period. As for the DOX/Z-NCs group, the DOX release pattern showed similar tendency. In brief, the DOX release was found to be $77.90 \%$ at $24 \mathrm{~h}, 80.37 \%$ at $36 \mathrm{~h}$, and after that, the percentage of released DOX was at $\sim 82 \%$. Comparatively, accelerated DOX release was not found in the other two groups. In the conditions of GSH $2.8 \mu \mathrm{M}$ and GSH 0, DOX release from DOX/FA-Z-NCs or DOX/Z-NCs showed relatively low release rate. In the initial $12 \mathrm{~h}$, the cumulative release was lower than $5 \%$. With increase in incubation time, release of accumulated DOX showed very slow growth. When the incubation time was extended to $72 \mathrm{~h}$, the cumulative DOX release only reached $\sim 12 \%$ in PBS and $\sim 17 \%$ in GSH $2.8 \mu \mathrm{m}$ conditions. It should be noted that, despite the fact that a GSH-accelerated drug release pattern from the nanocapsules was achieved, the maximum cumulative release was only $\sim 82 \%$. This incomplete drug release was also found in our previous research ${ }^{28}$ and that of others, ${ }^{35}$ where the electrostatic interaction between the positively charged DOX and the negatively charged building materials of nanocarriers retarded DOX release from the dialysis bag.

\section{In vitro cytotoxicity}

In vitro cytotoxicities of DOX/FA-NCs, DOX/Z-NCs, and free drug $\mathrm{DOX} \cdot \mathrm{HCl}$ against breast tumor $4 \mathrm{~T} 1$ cells were examined by CCK-8 assay. As shown in Figure 3, the viability of the 4T1 cells highly relied on the concentration of co-cultured DOX. At low DOX concentration $(\leq 0.001 \mu \mathrm{g} /$ $\mathrm{mL})$, the cells incubated with all the DOX-containing formulations exhibited a high $(>90 \%)$ cell viability. The commercial product $\mathrm{DOX} \cdot \mathrm{HCl}$ demonstrated the lowest cell viability, leading to $85.69 \%, 51.75 \%, 28.83 \%, 4.27 \%$ and

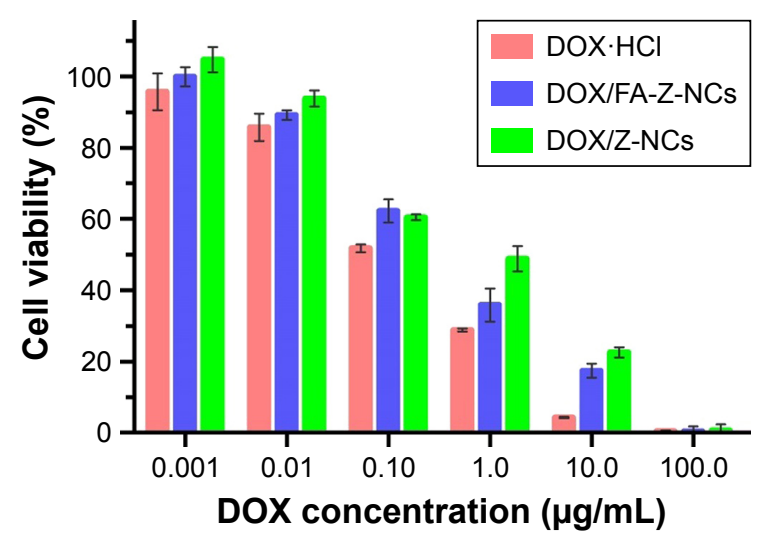

Figure 3 In vitro cytotoxicity assay.

Note: 4TI cells were incubated with DOX-containing formulations for $36 \mathrm{~h}$.

Abbreviations: DOX, doxorubicin; DOX.HCl, doxorubicin hydrochloride; FA, folic acid; NCs, nanocapsules.
$0.44 \%$ of viable $4 \mathrm{~T} 1$ cells at the DOX concentrations of 0.01 , $0.10,1.0,10.0$ and $100.0 \mu \mathrm{g} / \mathrm{mL}$, respectively. In comparison, the DOX-encapsulated nanocapsules showed slightly weakened tumor cell growth inhibition effect. When the DOX concentration was $0.1 \mu \mathrm{g} / \mathrm{mL}$, cell viability of the two DOX-encapsulated nanocapsules was still higher than $60 \%$. According to the cell viability data shown in Figure 3, the $\mathrm{IC}_{50}$ value was estimated to be $0.15,0.33$, and $0.60 \mu \mathrm{g} / \mathrm{mL}$ for DOX.HCl, DOX/FA-Z-NCs, and DOX/Z-NCs, respectively (data fitting was performed by using GraphPad Prism 5). The different in vitro tumor cell inhibition efficacies between the free drug and DOX/NCs suggested that release and diffusion of the DOX molecules in nanocapsules were limited by the nanocapsule shells, at least to some extent. Besides, cytocompatibility of the hollow nanocapsules was examined (Figure S8). Without drug encapsulation, these hollow nanocapsules showed high cytobiocompatibility $(>92 \%)$ even at the highest concentration $(809.091 \mu \mathrm{g} / \mathrm{mL}$ for FA-Z-NCs and $790.909 \mu \mathrm{g} / \mathrm{mL}$ for Z-NCs; concentrations of the hollow nanocapsules were referred to the concentrations of the corresponding drug-encapsulating nanocapsules used for cell viability assay) of the carrier materials. This result demonstrated that the nanocapsules, either FA-Z-NCs or Z-NCs, had very low toxicity to the tumor cell line, and the detected cytotoxicity effect should be attributed to the encapsulated DOX.

\section{Cellular uptake and endosomal escape}

Flow cytometry was used to analyze cellular uptake of the DOX-containing formulations and the corresponding fluorescent signal changes over time, as shown in Figure 4. Cellular uptake of these three samples (DOX.HCl, DOX/ FA-Z-NCs, and DOX/Z-NCs) increased markedly even after $2 \mathrm{~h}$ of co-culture (Figure 4A). Among them, DOX/ FA-Z-NCs and DOX/Z-NCs exhibited almost the same cellular uptake tendency, in contrast to the slightly lesser extent for DOX $\cdot \mathrm{HCl}$. When further exposed to $4 \mathrm{~T} 1$ cells for $24 \mathrm{~h}$ (Figure 4B), an increase in fluorescence intensity was observed in the case of DOX/FA-Z-NCs, demonstrating the cellular uptake was enhanced in comparison to the other two samples. Therefore, it could be concluded that cellular uptake of the nanocapsules could be markedly accelerated by introducing the targeting ligand FA. Moreover, this enhanced cellular uptake effect would be a reasonable explanation for the above-detected difference between their cytotoxic activities in spite of their similar drug encapsulation content (11\% for DOX/FA-Z-NCs and 13\% for DOX/Z-NCs) and equivalent drug concentration for tests (Figure 3 ). 
A

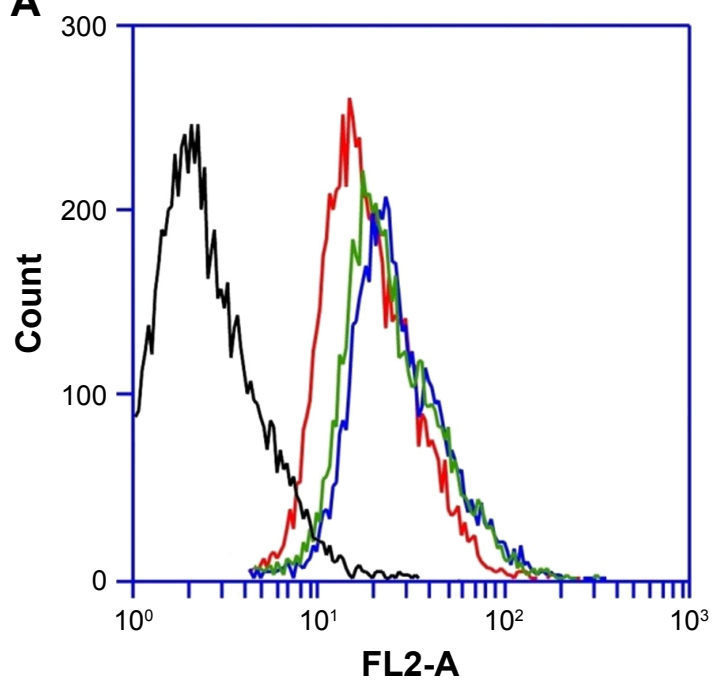

B

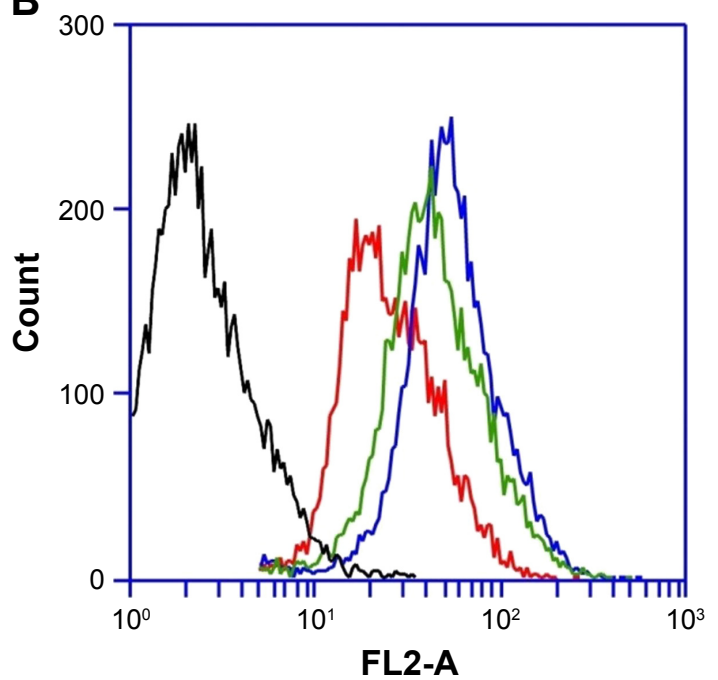

- Control — DOX.HCl — DOX/FA-Z-NCs — DOX/Z-NCs

Figure 4 Flow cytometry results of time-dependent DOX signal changes.

Note: (A) $2 \mathrm{~h}$ and (B) $24 \mathrm{~h}$.

Abbreviations: DOX, doxorubicin; DOX.HCl, doxorubicin hydrochloride; FL2-A, fluorescent signal of DOX; FA, folic acid; NCs, nanocapsules.

After endocytosis, it is necessary to avoid degradation before the DOX can be released into the cytosol to achieve its therapeutic effect. It was proposed that once the nanocapsules are disassembled by intracellular GSH, the swelling nanocapsule shells would help in disruption of the endolysosomal membrane and, thus, conduct efficient endosomal escape of the DOX. In order to verify this, CLSM measurements were applied and corresponding fluorescent signals of the DOX-containing formulations (DOX, DOX/ $\mathrm{NCs})$ and lysotracker-labeled intracellular acidic compartments (ie, endosomes and lysosomes) were obtained. As shown in Figure 5, we investigated the time-dependent DOX/nanocapsule transportation inside 4T1 cells, where the DOX or DOX/NCs (red), acidic endolysosomes (green), and their overlapped image (yellow/orange) were presented as specific fluorescent signal channels. In general, cellular trafficking of these three DOX-containing formulations was not synchronized. Particularly, DOX $\cdot \mathrm{HCl}$ showed the fastest internalization process. At $0.5 \mathrm{~h}$, internalized DOX $\cdot \mathrm{HCl}$ molecules were rapidly delivered to the nuclei. On the contrary, the encapsulated DOX, either in DOX/FA-Z-NCs or in DOX/Z-NCs group, was mostly monitored outside the nuclei at $0.5 \mathrm{~h}$. This could be explained by the fact that diffusion across the cell membrane and cellular uptake of DOX. $\mathrm{HCl}$ could be achieved easily. This result was in high accordance with our cytotoxicity test, where the diffusion/release of the encapsulated DOX was moderated by the nanocapsule shells (Figure 3), and a similar result was also found in our previous work. ${ }^{28}$ With increase in incubation time, DOX internalization became marked. For the DOX.HCl, DOX accumulation at the cytoplasm was enhanced from 0.5 to $6 \mathrm{~h}$, and most of the internalized DOX was located at the nuclei. As for the DOX/NCs groups, the overlap (yellow/orange images) of DOX channel and lysotracker channel became obvious and DOX fluorescence was gradually visible at the nuclei. These results indicated that transportation of the encapsulated DOX toward nuclei was limited by the nanocapsule shells, as compared with the rapidly diffusing DOX.HCl. It should be noted that incorporating the targeting ligand FA on HA nanocapsules speeded up the cellular uptake. As shown in the CLSM images (Figure 5, second and third columns), the orange/yellow domains could be observed clearly in DOX/ FA-Z-NCs group at $0.5 \mathrm{~h}$, while the DOX/Z-NCs group still showed quite a negative fluorescent signal. On increasing the incubation time to 2 and $6 \mathrm{~h}$, this difference between DOX/FA-Z-NCs and DOX/Z-NCs groups gradually became marked. These results verified the targeting effect of the FA, which facilitated internalization of DOX/FA-Z-NCs by the FA receptor overexpression in $4 \mathrm{~T} 1$ cells. ${ }^{36}$

\section{In vivo antitumor efficacy}

In an attempt to investigate the antitumor efficacy and related toxicity induced by the drug-containing formulations, tumor volume and body weight of each tumor-bearing BALB/c mouse after intravenous injections were recorded. As shown in Figure 6A, these changes were expressed as 

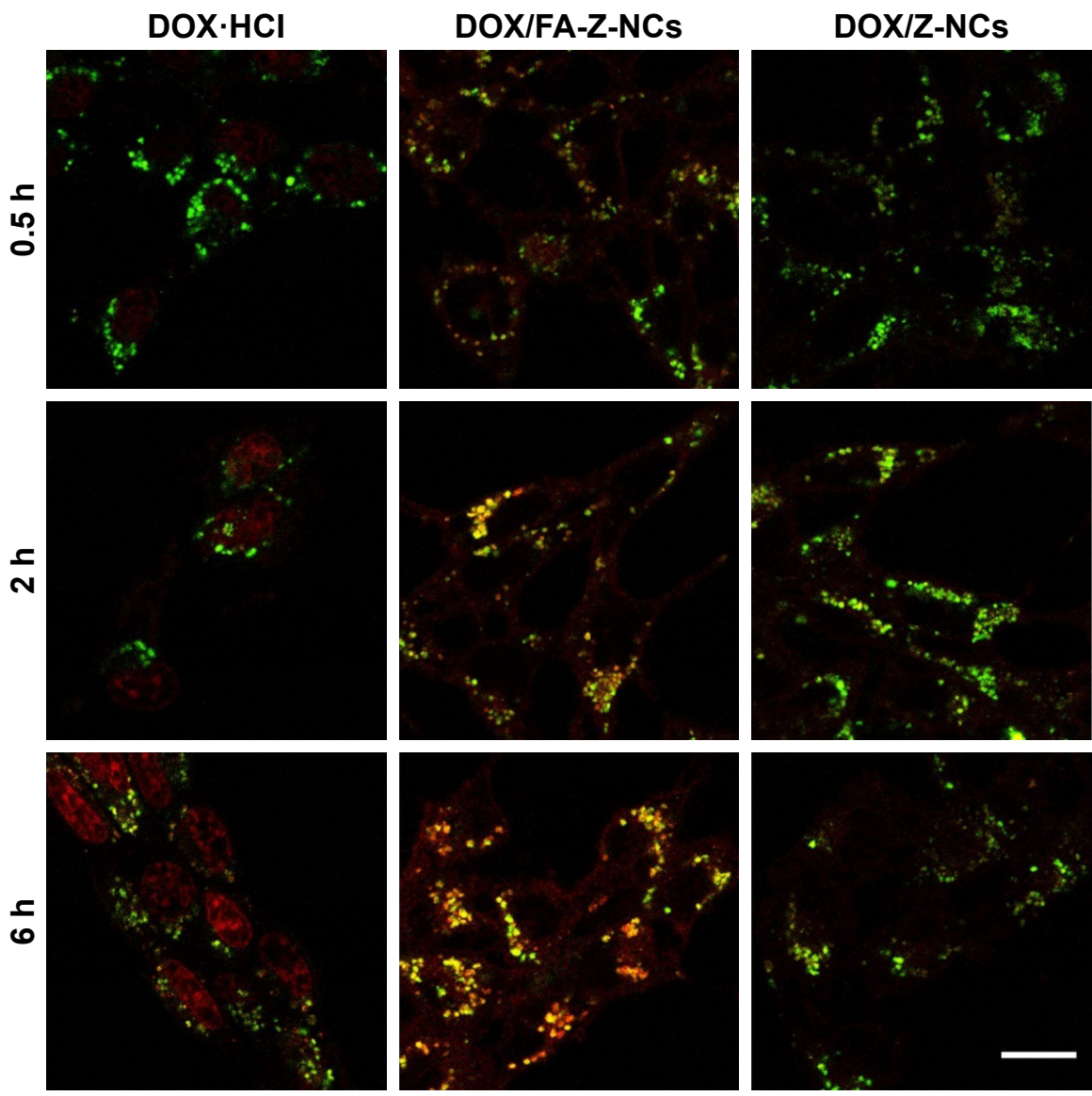

Figure 5 CLSM images of 4TI cell lines incubated with DOX.HCl, DOX/FA-Z-NCs, and DOX/Z-NCs for 0.5, 2, and $6 \mathrm{~h}$.

Notes: The fluorescent channels show signals of DOX (red), lysotracker green-labeled acidic endolysosomes (green), and the merged images (orange/yellow). Scale bar measures $20 \mu \mathrm{m}$.

Abbreviations: CLSM, confocal laser scanning microscopy; DOX, doxorubicin; DOX.HCl, doxorubicin hydrochloride; FA, folic acid; NCs, nanocapsules.

A

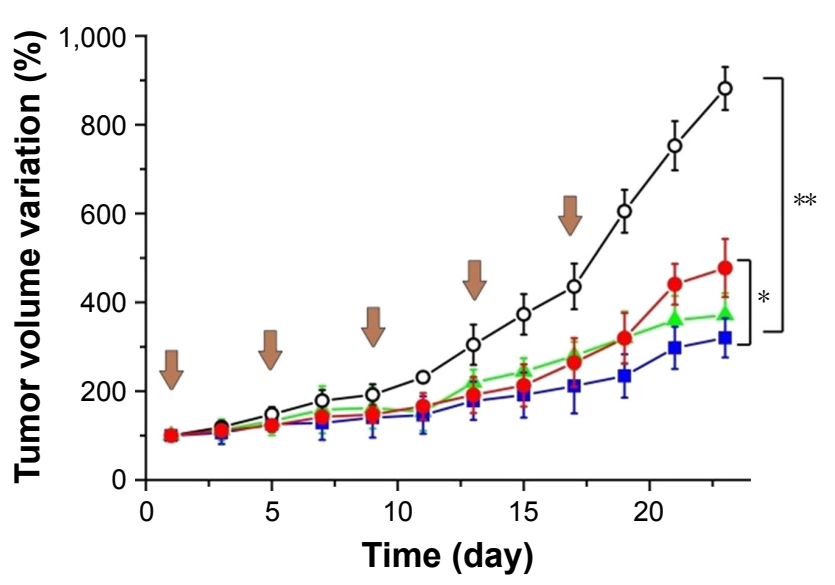

B

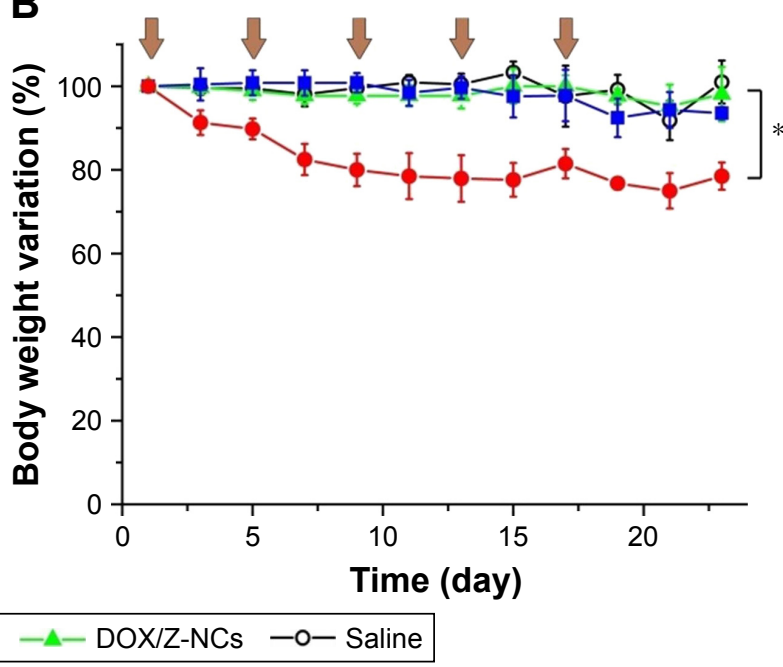

Figure 6 In vivo antitumor efficacy.

Notes: Variations in $(\mathbf{A})$ tumor volume and $(\mathbf{B})$ body weight of the tumor-bearing BALB/c mice $(n=5)$. In $(\mathbf{A})$, the DOX/FA-Z-NCs demonstrated significant tumor inhibition effect $\left({ }^{*} p<0.00\right.$ I, compared to saline; ${ }^{*} p<0.05$, compared to DOX.HCl). In $(\mathbf{B})$, the saline and DOX/NCs groups showed no significant effect on body weight, whereas the DOX $\cdot \mathrm{HCl}$ induced obvious body weight loss $(* p<0.05$, compared to DOX. HCl). The arrows represent all the five injections during the experimental period.

Abbreviations: DOX, doxorubicin; DOX. $\mathrm{HCl}$, doxorubicin hydrochloride; FA, folic acid; $\mathrm{NCs}$, nanocapsules. 
the relative increase percentages as compared with their initial values. In the tumor-bearing $\mathrm{BALB} / \mathrm{c}$ mice, treated with either free DOX (DOX. $\mathrm{HCl}$ ) or encapsulated DOX (in FA-Z-NCs or Z-NCs), their tumor volume increased as a function of time. Nonetheless, obvious differences among the four groups could be observed. Generally, the fastest tumor growth was found in the saline group. Without DOX treatment, the tumor size almost doubled $(192.0 \% \pm 23.6 \%)$ at day 9 , showed a $436.0 \% \pm 51.5 \%$ increase at day 16 , and became $881.7 \% \pm 48.3 \%$ at the end of experimental day (day 23). On the contrary, the DOX-containing formulations slowed tumor growth. At the last experimental day, the tumor volume increase percentages (\%) in various groups were found to be $320.15 \% \pm 44.22 \%$ for DOX/FA-Z-NCs, $371.2 \% \pm 49.2 \%$ for $\mathrm{DOX} / \mathrm{Z}-\mathrm{NCs}$, and $477.6 \% \pm 65.5 \%$ for $\mathrm{DOX} \cdot \mathrm{HCl}$. These results verified the antitumor effect of all the three DOX-containing formulations. Furthermore, these results also evidenced that the nanocapsules and their peripheral targeting ligand FA could promote the in vivo antitumor efficacy, as we found significant tumor inhibition effect of DOX/FA-Z-NCs $(* * p<0.001$, compared to saline; ${ }^{*} p<0.05$, compared to $\left.\mathrm{DOX} \cdot \mathrm{HCl}\right)$. Besides the tumor inhibition effect, the body weight of each mouse was carefully recorded, in order to monitor potential severe damage caused by injections. As shown in Figure 6B, the $\mathrm{DOX} \cdot \mathrm{HCl}$ group exhibited an obvious body weight loss during the experiment. Specifically, a $20 \%$ body weight loss was found at day 9 and $21.5 \%$ was found at the end of the experimental day. On the contrary, the other three groups (DOX/FA-Z-NCs, DOX/ZNCs, and saline) showed no significant body weight loss. These results suggested that the nanocapsules possessed good biocompatibility and the encapsulated DOX would endow a rather low degree of systemic toxicity to the mice.

\section{Biodistribution study}

In order to study possible in vivo distribution of these DOXcontaining formulations, ex vivo imaging was performed to monitor DOX signals in the excised major organs and tumor tissues at different time intervals $(2,7$, and $24 \mathrm{~h}$ ) postadministration (Figure 7). Fluorescent signals of the three sample groups were visualized in the tumor tissues $2 \mathrm{~h}$ after injection, which indicated that DOX could accumulate in the tumors rapidly. After that, these signals showed a gradual increase in tumors and kidneys and a decrease in livers, demonstrating clearance of DOX by the liver during blood circulation. ${ }^{37}$ It was interesting to know that, the strongest fluorescent signal in tumor sites was found in the DOX/Z-NCs group at $7 \mathrm{~h}$. Thus, one might argue the potential therapeutic effect associated with the targeting ligand FA. Considering the clinical practice, where multiple injections are favored for tumor treatment, comprehensive investigations into tumor growth inhibition and acute systemic toxicity caused by accumulation of the therapeutic agents within the effective time window are of great importance. For this reason, DOX accumulation in the major organs and tumors over $24 \mathrm{~h}$ was studied. Among the three samples, DOX/FA-Z-NCs group exhibited the fastest clearance, proven by the high amount of DOX deposited (average signal 789.71 \pm 21.27 , counts) in the tumor and nearly zero signal (average signal $34.83 \pm 4.87$, counts) in the kidneys at $24 \mathrm{~h}$ after injection. It was noteworthy that ex vivo fluorescence signal of heart was obvious in the DOX $\cdot \mathrm{HCl}$ group, and the average signal was $183.42 \pm 5.29$ (counts) even $24 \mathrm{~h}$ postadministration. This result indicated that the free $\mathrm{DOX} \cdot \mathrm{HCl}$ possessed significant potential to harm the heart, although its accumulation at tumor sites was detectable (average signal 329.32 \pm 22.50 , counts) after $24 \mathrm{~h}$. As for the DOX/Z-NCs, even though accumulation at the tumor site was considerable (average signal 601.19 \pm 10.62 , counts) after $24 \mathrm{~h}$, their accumulation in kidney $(550.65 \pm 55.00$, counts) and liver (682.28 \pm 5.64 , counts) was still obvious, indicating possible increased burden for these two organs. Therefore, these results again proposed that the FA-Z-NCs could serve as safe nanocarriers to deliver anticancer drugs/therapeutic agents for tumor therapy by maintaining suitable drug concentrations at the tumors and decreasing possible side effects to other major organs.

\section{Immunohistochemical studies of the tumors}

In order to investigate the mechanism of antitumor efficacy caused by DOX-containing formulations, immunohistochemical studies, Ki 67 antigen staining and transferasemediated deoxyuridine triphosphate-biotin nick-end labelling (TUNEL) assay, were conducted, as shown in Figure 8. Ki 67 staining was used to evaluate proliferation of the 4T1 cells. Optical densities (of the Ki 67-positive areas) of the $\mathrm{DOX} \cdot \mathrm{HCl}, \mathrm{DOX} / \mathrm{Z}-\mathrm{NCs}$, and DOX/FA-Z-NCs groups were $0.281 \pm 0.020,0.291 \pm 0.014$, and $0.268 \pm 0.023$, respectively. No statistical difference among these three groups was detected, which demonstrated that growth inhibition of 4T1 cells in tumor was roughly identical after treatment with the three samples. On the contrary, without drug treatment, the saline group showed a higher optical density $(0.339 \pm 0.035)$, demonstrating lower tumor cell growth inhibition percentage. On the other hand, the TUNEL assay was used to assess apoptotic programmed cell death. The saline group showed 

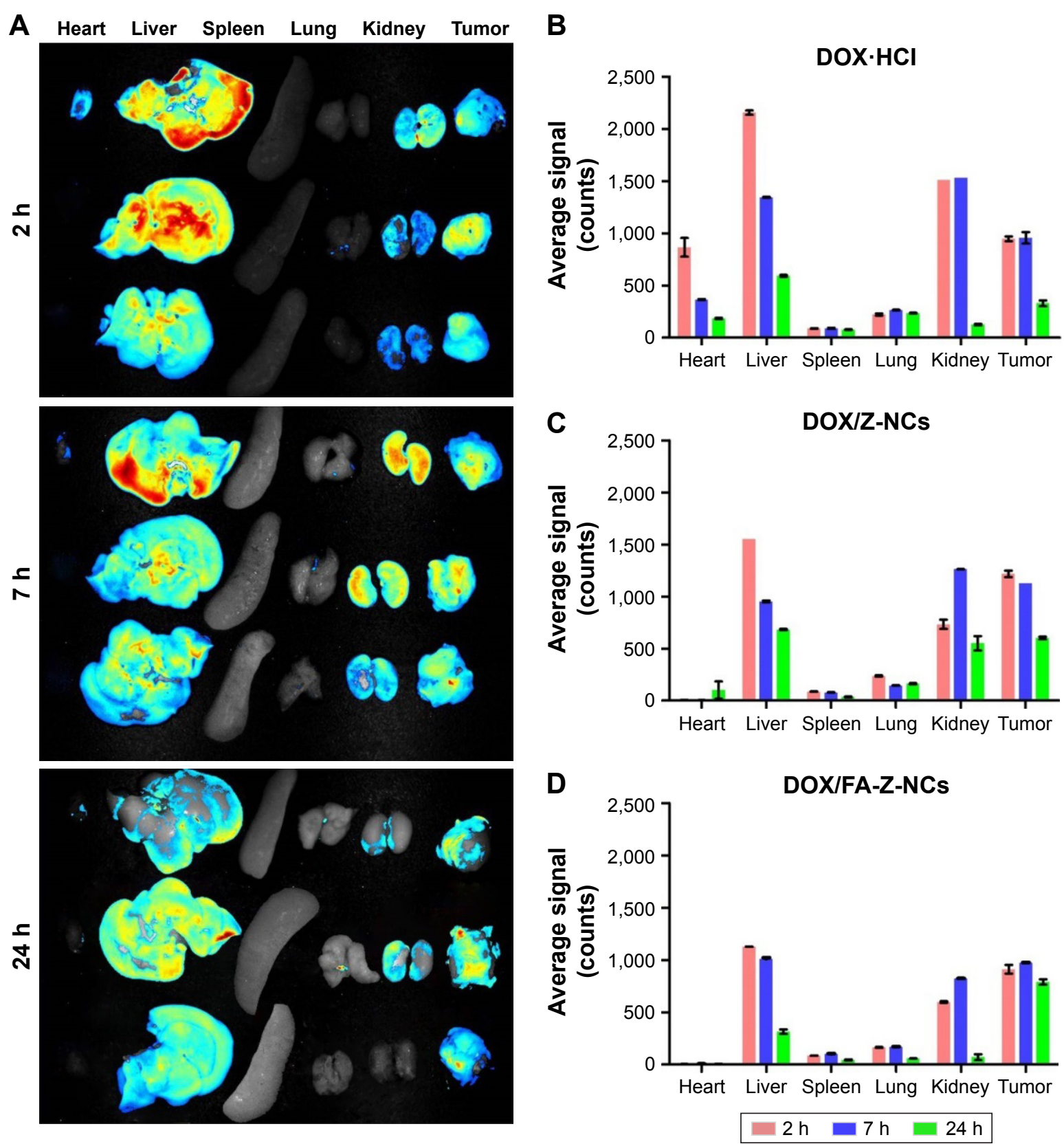

Figure 7 The $(\mathbf{A})$ ex vivo fluorescence images and $(\mathbf{B})$ average signals of excised major organs and tumor tissue at different time intervals $(2,7$, and $24 \mathrm{~h})$ postadministration in (B) DOX.HCl, (C) DOX/Z-NCs and (D) DOX/FA-Z-NCs groups.

Notes: The fluorescent signals were collected based on emission of the DOX, and the dosage of the injected DOX was normalized to be $5 \mathrm{mg} / \mathrm{kg}$. The sample groups were placed in the order DOX.HCl, DOX/Z-NCs, and DOX/FA-Z-NCs in each panel. Average signals were collected by using the Maestro in vivo imaging system. Abbreviations: DOX, doxorubicin; DOX.HCl, doxorubicin hydrochloride; FA, folic acid; NCs, nanocapsules.

the smallest TUNEL-positive areas $(9.0 \% \pm 3.8 \%)$, where the degree of $4 \mathrm{~T} 1$ cell apoptosis was the least. However, the other three groups showed a significant cell death $(p<0.05)$. In particular, the DOX/FA-Z-NCs exhibited the highest TUNEL-positive percentage of $51.0 \% \pm 7.3 \%$, compared with $38.0 \% \pm 6.4 \%$ for DOX/Z-NCs and $45.0 \% \pm 3.2 \%$ for DOX.HCl. Taken together, the immunohistochemical studies revealed that effective antitumor effect could be achieved through inhibition of tumor cell proliferation and induction of tumor cell apoptosis in situ; moreover, the DOX/FA-Z-NCs played the most significant role in influencing these parameters, offering vast potential to promote tumor suppression and further prognosis.

\section{Histologic studies of the major organs}

Hematoxylin and Eosin (HE) staining of the tissues of major organs was studied, giving further evidence of how the DOX-containing formulations affect normal 

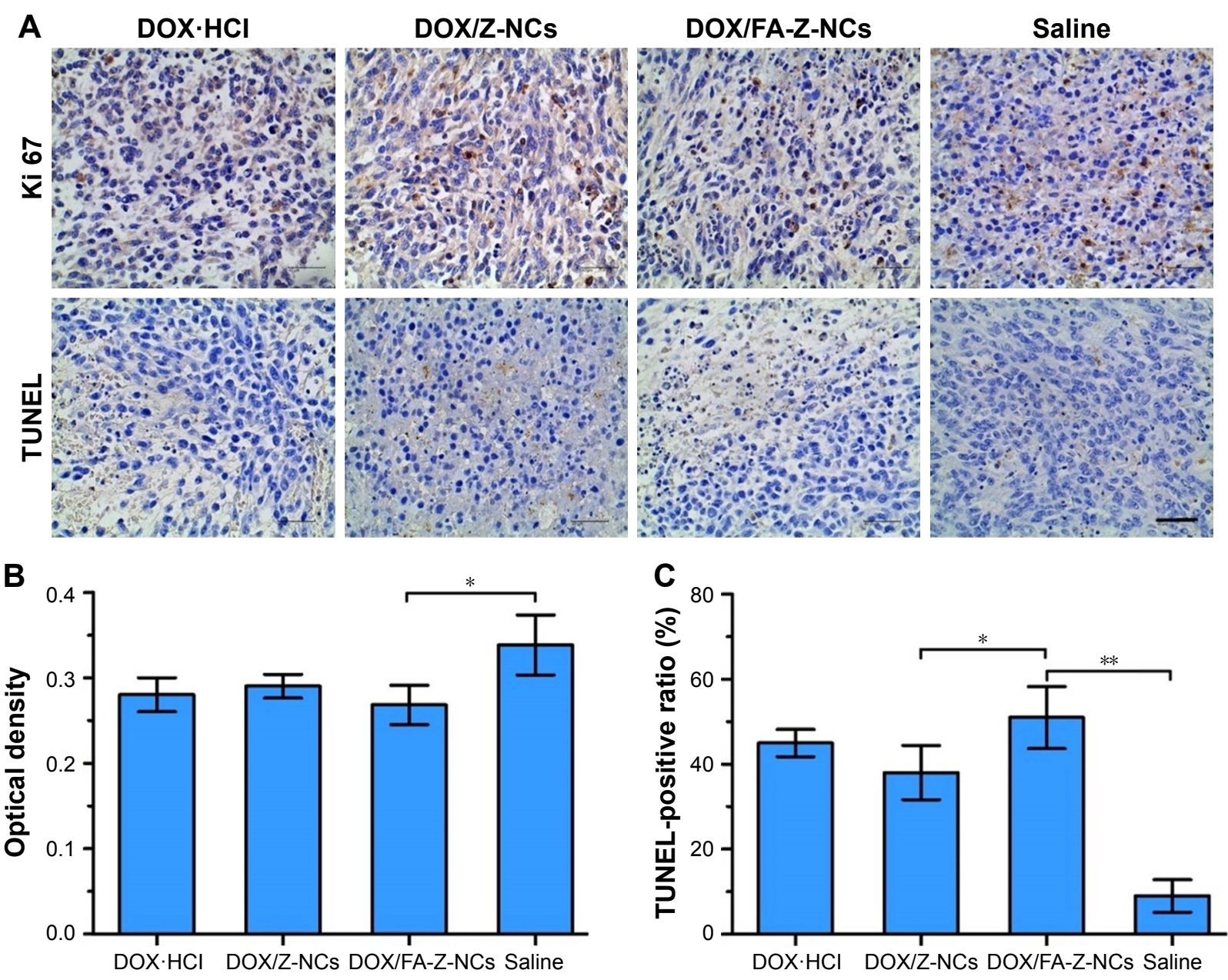

Figure 8 Immunohistochemical staining of tumor tissues.

Notes: (A) Ki 67 positive (brown) and TUNEL positive (brown) represent tumor cell proliferation inhibition and tumor apoptosis, respectively. Quantification of the (B) Ki 67-positive areas and (C) TUNEL-positive areas. In (A), scale bar measures $40 \mu \mathrm{m}$. In (B and C), the DOX-containing formulations exhibited significant tumor cell death $\left(* p<0.05\right.$, ${ }^{* *} p<0.00$ I).

Abbreviations: DOX, doxorubicin; DOX.HCl, doxorubicin hydrochloride; FA, folic acid; NCs, nanocapsules; TUNEL, transferase-mediated deoxyuridine triphosphatebiotin nick-end labelling.

physiological structures and possible malignant tumor metastasis, as shown in Figure 9. In this work, multiple injections of DOX $\cdot \mathrm{HCl}$ resulted in slight damage (+) to the heart, as proven by the granular degeneration of the cardiac muscles (Figure 9, first row and first column). Besides, moderate damage (++) of laminar necrosis in the liver, where some cells could be determined as invading tumor cells, was observed (Figure 9, second row and first column). Considering the in vivo antitumor efficacy (discussed above, Figure 6), it was observed that the free drug DOX $\cdot \mathrm{HCl}$ caused side effects to healthy normal organs and could not inhibit tumor metastasis, although it showed tumor growth inhibition ability to a certain extent. As for the saline group, diffuse hepatic necrosis and nucleus dissolution necrosis in the liver (Figure 9, second row and fourth column) and inflammatory cell infiltration in the lung (Figure 9, fourth row and fourth column) were found; moreover, tumor cell metastasis into the lung was also observed (Figure 9, fourth row and fourth column). In contrast, no obvious side effects of DOX or tumor metastasis could be found in the DOX/FA-Z-NCs and DOX/Z-NCs groups (Figure 9, third row and fourth row). These results again illustrated that the nanocapsules could not only serve as steady carriers for tumor-oriented drug delivery to avoid systematic toxicity but also enhance the efficacy of the therapeutic agents to inhibit tumor metastasis consequently.

\section{Conclusion}

The nanocapsule system DOX/FA-Z-NCs was fabricated and used for delivery of the antitumor drug DOX for tumor therapy. The nanocapsule shells were built up with crosslinked HA molecules and their tentacles were composed of the FA terminated zwitterionic polymers (pCBMA). Redispersing the dehydrated nanocapsules in water led to 

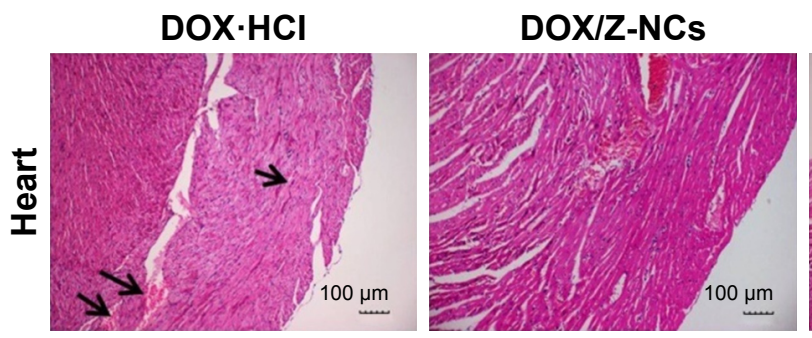

DOX/FA-Z-NCs
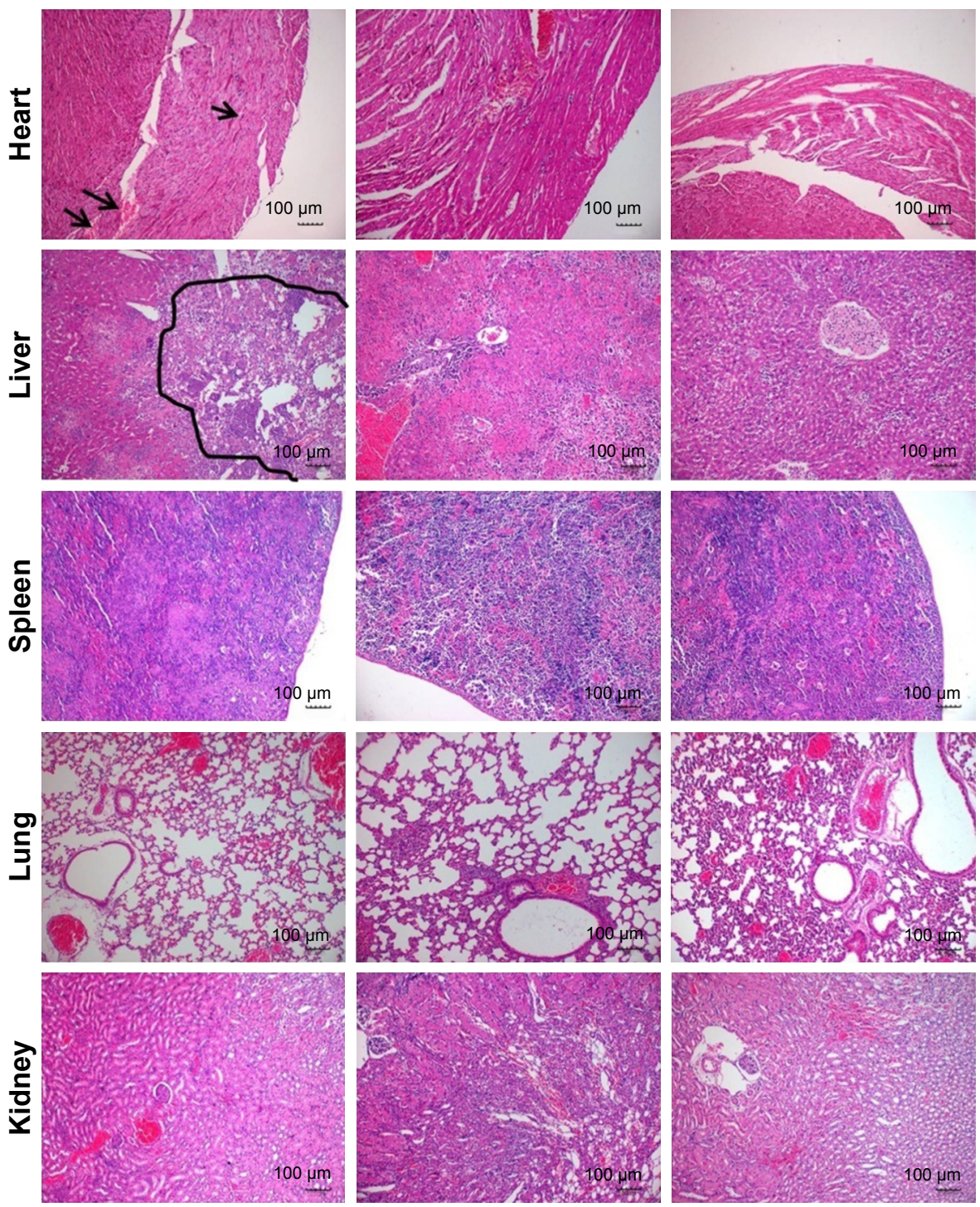
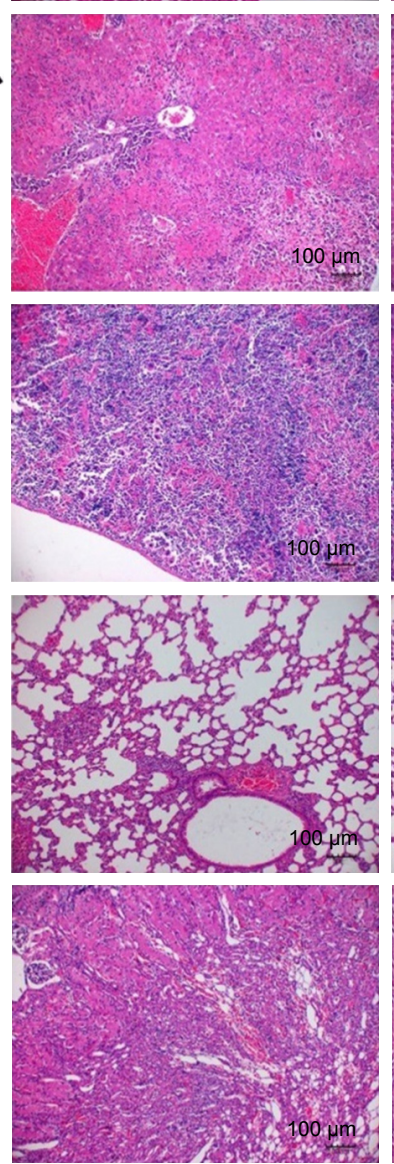
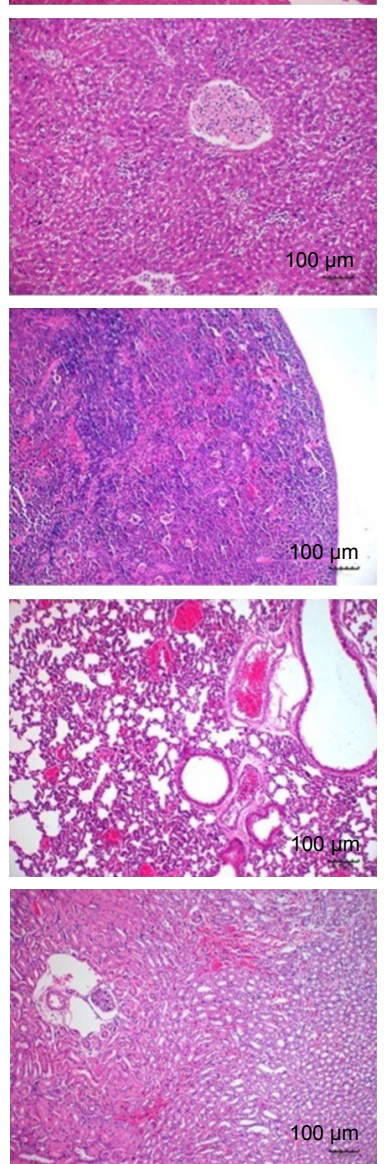

Saline
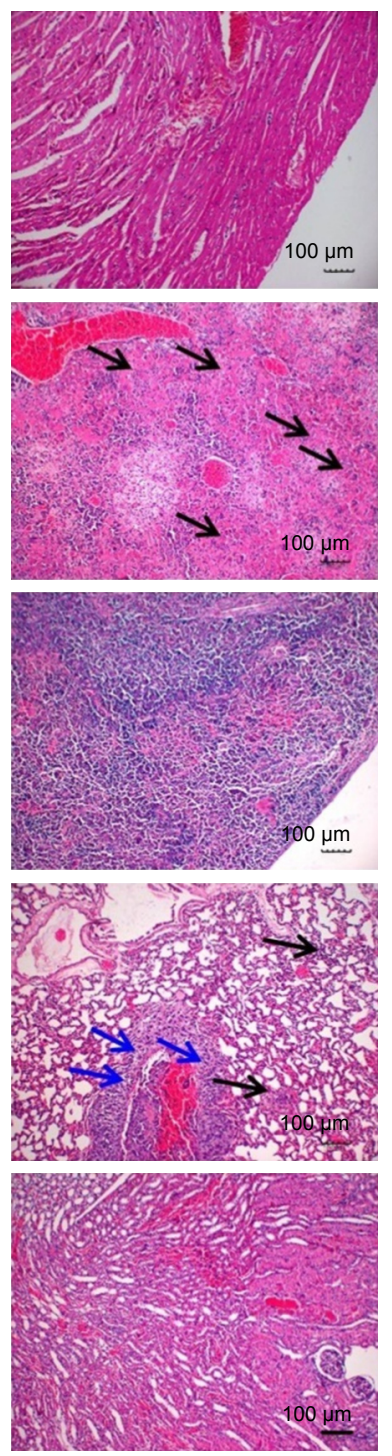

Figure 9 HE staining of tissues of major organs (heart, liver, spleen, lung, kidney; $\times 100$ ).

Notes: For the DOX.HCl group: black arrows point out granular degeneration of the cardiac muscles and the black line marks laminar necrosis of liver cells. For the saline group: the black arrows point out diffuse hepatic necrosis and nucleus dissolution necrosis in the liver and inflammatory cell infiltration in the lung; the blue arrows point out tumor cell metastasis into the lung. Scale bar measures $100 \mu \mathrm{m}$.

Abbreviations: DOX, doxorubicin; DOX.HCl, doxorubicin hydrochloride; FA, folic acid; HE, Hematoxylin and Eosin; NCs, nanocapsules.

a four-fold volume increase. These hydrogel-like shells served as large cavities for safe drug storage and a DOX encapsulation content of $11 \%$ was achieved. In vitro experiments verified that the targeting ligand FA enhanced cytotoxicity and cellular uptake. Intracellular bioreduction environment (high GSH level inside 4T1 cells) resulted in the escape of nanocapsules from the endosomes and governed release of DOX into the cytosol, benefiting from the GSH-triggered nanocapsule shell swelling and disassembly. In vivo evaluations demonstrated effective antitumor capability as well as greatly reduced side effects to normal major organs of the DOX/FA-Z-NCs due to their selective accumulation in tumor sites via an FA-conducted active delivery mechanism.

\section{Acknowledgments}

Financial support from the National Natural Science Foundation of China (81471775, 81361140343, and 81621003), Science and Technology Research Program of Sichuan Provence (2017HH0006), and Fundamental Research Funds for the Central Universities (2015SCU04A31) is greatly appreciated.

\section{Disclosure}

The authors report no conflicts of interest in this work. 


\section{References}

1. Cho K, Wang X, Nie S, Chen ZG, Shin DM. Therapeutic nanoparticles for drug delivery in cancer. Clin Cancer Res. 2008;14:1310-1316.

2. Shi J, Votruba AR, Farokhzad OC, Langer R. Nanotechnology in drug delivery and tissue engineering: from discovery to applications. Nano Letters. 2011;10:3223-3230.

3. Savić R, Azzam T, Eisenberg A, Maysinger D. Assessment of the integrity of poly(caprolactone)-b-poly(ethylene oxide) micelles under biological conditions: a fluorogenic-based approach. Langmuir. 2006; 22:3570-3578.

4. Alexis F, Pridgen E, Molnar LK, Farokhzad OC. Factors affecting the clearance and biodistribution of polymeric nanoparticles. Mol Pharm. 2008;5:505-515.

5. Stuart MA, Huck WT, Genzer J, et al. Emerging applications of stimuliresponsive polymer materials. Nat Mater. 2010;9:101-113.

6. Sivakumar S, Bansal V, Cortez C, Chong SF, Zelikin AN, Caruso F. Degradable, surfactant-free, monodisperse polymer-encapsulated emulsions as anticancer drug carriers. Adv Mater. 2009;21: 1820-1824.

7. Johnston AP, Lee L, Wang Y, Caruso F. Controlled degradation of DNA capsules with engineered restriction-enzyme cut sites. Small. 2009; 5(12):1418-1421.

8. Zhen S, Wang C, Li J, Sukhorukov GB, Antipina MN. Encapsulation of basic fibroblast growth factor by polyelectrolyte multilayer microcapsules and its controlled release for enhancing cell proliferation. Biomacromolecules. 2012;13(7):2174-2180.

9. Liang K, Such GK, Johnston AP, et al. Endocytic pH-triggered degradation of nanoengineered multilayer capsules. Adv Mater. 2014; 26(12):1901-1905.

10. Gao H, Wen D, Tarakina NV, Liang J, Bushby AJ, Sukhorukov GB. Bifunctional ultraviolet/ultrasound responsive composite $\mathrm{TiO}_{2} /$ polyelectrolyte microcapsules. Nanoscale. 2016;8:5170-5180.

11. Yi Q, Sukhorukov GB. UV light stimulated encapsulation and release by polyelectrolyte microcapsules. Adv Colloid Interface Sci. 2014; 207:280-289.

12. Pavlov AM, De Geest BG, Louage B, et al. Magnetically engineered microcapsules as intracellular anchors for remote control over cellular mobility. Adv Mater. 2013;25(48):6945-6950.

13. Semmling M, Kreft O, Muñoz JA, Sukhorukov GB, Käs J, Parak WJ. A novel flow-cytometry-based assay for cellular uptake studies of polyelectrolyte microcapsules. Small. 2008;4(10):1763-1768.

14. Yi Q, Li D, Lin B, et al. Magnetic resonance imaging for monitoring of magnetic polyelectrolyte capsule in vivo delivery. BioNanoScience. 2014;4(1):59-70.

15. Danhier F. To exploit the tumor microenvironment: since the EPR effect fails in the clinic, what is the future of nanomedicine? J control Release. 2016;244:108-121.

16. Zha L, Banik B, Alexis F. Stimulus responsive nanogels for drug delivery. Soft Matter. 2011;7:5908-5916.

17. Wauthoz N, Bastiat G, Moysan E, et al. Safe lipid nanocapsule-based gel technology to target lymph nodes and combat mediastinal metastases from an orthotopic non-small-cell lung cancer model in SCID-CB17 mice. Nanomedicine. 2015;11(5):1237-1245.

18. Lee Y, Park SY, Kim C, Park TG. Thermally triggered intracellular explosion of volume transition nanogels for necrotic cell death. J Controlled Release. 2009;135:89-95.

International Journal of Nanomedicine

\section{Publish your work in this journal}

The International Journal of Nanomedicine is an international, peerreviewed journal focusing on the application of nanotechnology in diagnostics, therapeutics, and drug delivery systems throughout the biomedical field. This journal is indexed on PubMed Central, MedLine, CAS, SciSearch $\AA$, Current Contents ${ }^{\circledR} /$ Clinical Medicine,
19. Bae KH, Lee Y, Park TG. Oil-encapsulating PEO-PPO-PEO/PEG shell cross-linked nanocapsules for target-specific delivery of paclitaxel. Biomacromolecules. 2007;8(2):650-656.

20. Bartolazzi A, Peach R, Aruffo A, Stamenkovic I. Interaction between CD44 and hyaluronate is directly implicated in the regulation of tumor development. J Exp Med. 1994;180(1):53-66.

21. Son S, Namgung R, Kim J, Singha K, Kim WJ. Bioreducible polymers for gene silencing and delivery. Acc Chem Res. 2012;45(7):1100-1112.

22. Ulbrich K, Holá K, Šubr V, Bakandritsos A, Tuček J, Zbořil R. Targeted drug delivery with polymers and magnetic nanoparticles: covalent and noncovalent approaches, release control, and clinical studies. Chem Rev. 2016;116(9):5338-5431.

23. Ross JF, Chaudhuri PK, Ratnam M. Differential regulation of folate receptor isoforms in normal and malignant tissues in vivo and in established cell lines. Physiologic and clinical implications. Cancer. 1994;73(9):2432-2443.

24. Nicolas J, Mura S, Brambilla D, Mackiewicz N, Couvreur P. Design, functionalization strategies and biomedical applications of targeted biodegradable/biocompatible polymer-based nanocarriers for drug delivery. Chem Soc Rev. 2013;42(3):1147-1235.

25. Ishida T, Masuda K, Ichikawa T, Ichihara M, Irimura K, Kiwada $H$. Accelerated clearance of a second injection of PEGylated liposomes in mice. Int J Pharm. 2003;255(1-2):167-174.

26. Ma J, Kang K, Yi Q, Zhang Z, Gu Z. Multiple $\mathrm{pH}$ responsive zwitterionic micelles for stealth delivery of anticancer drugs. RSC Adv. 2016; 69:64778-64790.

27. SchlenoffJB. Zwitteration: coating surfaces with zwitterionic functionalityto reduce nonspecific adsorption. Langmuir. 2014;30(32):9625-9636.

28. Yi Q, Ma J, Kang K, Gu Z. Dual cellular stimuli-responsive hydrogel nanocapsules for delivery of anticancer drugs. JMater Chem B. 2016;4: $4922-4933$.

29. Bencherif SA, Srinivasan A, Horkay F, Hollinger JO, Matyjaszewski K, Washburn NR. Influence of the degree of methacrylation on hyaluronic acid hydrogels properties. Biomaterials. 2008;29(12):1739-1749.

30. Lee ES, Na K, Bae YH. Polymeric micelle for tumor $\mathrm{pH}$ and folatemediated targeting. J Control Release. 2003;91(1-2):103-113.

31. Wang Y, Yu L, Han L, Sha X, Fang X. Difunctional Pluronic copolymer micelles for paclitaxel delivery: synergistic effect of folate-mediated targeting and Pluronic-mediated overcoming multidrug resistance in tumor cell lines. Int J Pharm. 2007;337(1-2):63-73.

32. Prata JE, Barth TA, Bencherif SA, Washburn NR. Complex fluids based on methacrylated hyaluronic acid. Biomacromolecules. 2010;11(3): 769-775.

33. Leach JB, Bivens KA, Patrick CW Jr, Schmidt CE. Photocrosslinked hyaluronic acid hydrogels: natural, biodegradable tissue engineering scaffolds. Biotechnol Bioeng. 2003;82(5):578-589.

34. Wen HY, Dong HQ, Xie WJ, et al. Rapidly disassembling nanomicelles with disulfide-linked PEG shells for glutathione-mediated intracellular drug delivery. Chem Commun (Camb). 2011;47(12):3550-3552.

35. Pan YJ, Chen YY, Wang DR, et al. Redox/pH dual stimuli-responsive biodegradable nanohydrogels with varying responses to dithiothreitol and glutathione for controlled drug release. Biomaterials. 2012;33(27):6570-6579.

36. Kim D, Lee ES, Park K, Kwon IC, Bae YH. Doxorubicin loaded pHsensitive micelle: antitumoral efficacy against ovarian A2780/DOXR tumor. Pharm Res. 2008;25(9):2074-2082.

37. Li N, Yi Q, Luo K, Guo C, Pan D, Gu Z. Amphiphilic peptide dendritic copolymer-doxorubicin nanoscale conjugate self-assembled to enzymeresponsive anti-cancer agent. Biomaterials. 2014;35(35):9529-9545.

\section{Dovepress}

Journal Citation Reports/Science Edition, EMBase, Scopus and the Elsevier Bibliographic databases. The manuscript management system is completely online and includes a very quick and fair peer-review system, which is all easy to use. Visit http://www.dovepress.com/ testimonials.php to read real quotes from published authors. 